Annals of Pure and Applied Mathematics

Vol. 14, No. 1, 2017, 77-85

ISSN: 2279-087X (P), 2279-0888(online)

Published on 10 July 2017

Annals of

www.researchmathsci.org

DOI: http://dx.doi.org/10.22457/apam.v14n1a10

Pure and Applied

Mathematics

\title{
Fixed Point Theorems for Kannan Contractions and Weakly Contractive Mappings on a Modular Metric Space Endowed with a Graph
}

\author{
Prerna Pathak ${ }^{1}$, Aklesh Pariya ${ }^{2}$, V. H. Badshah ${ }^{3}$ and Nirmala Gupta ${ }^{4}$ \\ ${ }^{1,3}$ School of Studies in Mathematics Vikram University, Ujjain (M.P.), India \\ Email: prernapathak20@yahoo.com \\ ${ }^{2}$ Department of Mathematics, Medi-caps University, Indore (M.P.), India \\ Email: akleshpariya3@yahoo.co.in \\ ${ }^{4}$ Department of Mathematics, Govt. Girls Degree College, Ujjain (M.P.), India \\ Email:gupta.nirmala70@gmail.com \\ Corresponding author. Email: prernapathak20@yahoo.com
}

Received 13 June 2017; accepted 29 June 2017

Abstract. The notion of a modular metric spaces were introduced by Chistyakov [5, 6]. Abdou and Khamsi [1] gave the analog of Banach contraction principle in modular metric spaces. More recently, Alfuraidan [3] gave generalization of Banach contraction principle on a modular metric space endowed with a graph which is the modular metric version of Jachymski [8] fixed point results.

In this paper, we generalize and prove some fixed point results for Kannan contraction and weakly contractive mappings in a modular metric space endowed with a graph. The result of this paper is new and improving the previously known result in modular metric spaces endowed with a graph.

Keywords: Modular metric spaces, common fixed point, connected graph, Banach contraction, Kannan contraction.

AMS Mathematics Subject Classification (2010): 47H09, 46B20, 47H10, 47E10

\section{Introduction}

The existence of fixed points for single valued mappings in partially ordered metric spaces was initially considered by Ran and Reurings [15]. Fixed point theorems for monotone single valued mappings in a metric space endowed with a partial ordering have been widely investigated. Recently, many results appeared giving sufficient condition for $f$ to be a $\mathrm{PO}$ if $(\mathrm{X}, \mathrm{d})$ is endowed with a partial ordering $\preccurlyeq$. These results are the hybrid of two fundamental and useful theorems in fixed point theory, Banach Contraction Principle and the Knaster-Tarski theorem (see[7]). Jachymski [8] obtain some useful result for mappings defined on a complete metric spaces endowed with a graph instead of partial ordering. Bojor [4] proved fixed point result for Kannan mappings in metric spaces endowed with a graph. Samreen and Kamran [16] proved fixed point theorems for 
Prerna Pathak, Aklesh Pariya, V. H. Badshah and Nirmala Gupta

weakly contractive mappings on a metric space endowed with a graph. After that many researchers have investigated in this direction by weakly contractive condition and analyzing connectivity condition of graph.

The notion of modular spaces was introduce by Nakano [13] and was intensively develop by Koshi and Shimogaki [11], Yamamuro [17] and by Musielak and Orlicz [12]. Recently, Aghanians and Nourozi [2] discuss the existence and uniqueness of the fixed point for Banach and Kannan contraction defined on modular spaces endowed with a graph.

The notion of a modular metric spaces was introduced by Chistyakov [5,6]. Further Abdou and Khamsi [1] gave the analog of Banach contraction principle in modular metric spaces. More recently, Alfuraidan [3] gave generalization of Banach contraction principle on a modular metric space endowed with a graph which is the modular metric version of Jachymski [8] fixed point results for mappings on a metric space with a graph.

Ran and Reurings [15] proved the following fixed points result.

Theorem 1.1. [15] Let $(X, \preccurlyeq)$ be a partially ordered set such that every pair $x, y \in X$ has an upper and lower bound. Let $\mathrm{d}$ be a metric on $\mathrm{X}$ such that $(\mathrm{X}, \mathrm{d})$ is a complete metric space. Let $f: X \rightarrow X$ be a continuous monotone (either order preserving or order reversing) mapping. Suppose that the following condition hold:

(1) There exist a $k \epsilon(0,1)$ with

$$
d(f(x), f(y)) \leqslant k d(x, y), \quad \text { for all } x \geqslant y .
$$

(2) There exist an $x_{0} \in X$ with $x_{0} \leqslant f\left(x_{0}\right)$ or $x_{0} \geqslant f\left(x_{0}\right)$.

Then $\mathrm{f}$ is a Picard operator (PO), that is, $\mathrm{f}$ has a unique fixed point $x_{*} \in X$ and for each $x \in X, \lim _{n \rightarrow \infty} f^{n} x=x_{*}$.

Nieto et al. in [14], proved the following fixed point theorem.

Theorem 1.2. [14] Let $(X, d)$ be a complete metric spaces endowed with a partial ordering $\leqslant$.Let $f: X \rightarrow X$ be an order preserving mapping such that there exists a $k \epsilon[0,1)$ with

$$
d(f(x), f(y)) \leqslant k d(x, y), \quad \text { for all } x \geqslant y .
$$

Assume that one of the following conditions holds:

(1) $f$ is continuous and there exists an $x_{0} \in X$ with $x_{0} \leqslant f\left(x_{0}\right)$ or $x_{0} \geqslant f x_{0}$;

(2) $(\mathrm{X}, \mathrm{d}, \preccurlyeq)$ is such that for any non decreasing $\left(x_{n}\right)_{n \in N}$, if $x_{n} \rightarrow x$, then $x_{n} \leqslant x$ for $n \in N$, and there exist an $x_{0} \in X$ with $x_{0} \preccurlyeq f\left(x_{0}\right)$;

(3) $(\mathrm{X}, \mathrm{d}, \preccurlyeq)$ is such that for any non-decreasing $\left(x_{n}\right)_{n \in N}$, if $x_{n} \rightarrow x$, then $x_{n} \geqslant x$ for $n \in N$, and there exist an $x_{0} \in X$ with $x_{0} \geqslant f\left(x_{0}\right)$;

then $f$ has a fixed point. Moreover, if $(X, \preccurlyeq)$ is such that every pair of elements of $X$ has an upper or a lower bound, then $\mathrm{f}$ is a PO.

Jachymski [9] obtained the contraction principle for mappings on a metric spaces endowed with a graph.

Theorem 1.3. [9] Let $(X, d)$ be a complete metric spaceand let the triplet $(X, d, G)$ have the following property: 
Fixed Point Theorems for Kannan Contractions and Weakly Contractive Mappings on a Modular Metric Space Endowed with a Graph

(P) for any sequence $\left(x_{n}\right)_{n \in N}$ in $\mathrm{X}$ as $n \rightarrow \infty$ and $\left(x_{n}, x_{n+1}\right) \epsilon E(G)$, then $\left(x_{n}, x\right) \epsilon E(G)$, for all $\mathrm{n}$. Let $f: X \rightarrow X$ be a G-contraction. Then the following statements hold:

(1) $F_{f} \neq \varnothing$ if and only if $X_{f} \neq \emptyset$;

(2) if $X_{f} \neq \varnothing$ and G s weakly connected, then $\mathrm{f}$ is a Picard operator, i.e. $F_{f}=\left\{x^{*}\right\}$ and sequence $\left\{f^{n}(x)\right\} \rightarrow x^{*}$ as $n \rightarrow \infty$, for all $x \in X$;

(3) for any $x \in X_{f},\left.f\right|_{[x]_{\tilde{G}}}$ is a Picard operator ;

(4) if $X_{f} \subseteq E(G)$, then $\mathrm{f}$ is a weakly Picard operator, i.e., $F_{f} \neq \emptyset$ and, for each $x \in X$, we have a sequence $\left\{f^{n}(x)\right\} \rightarrow x^{*}(x) \in F_{f}$ as $n \rightarrow \infty$.

Bojor [4] proved fixed points of Kannan mappings in metric spaces endowed with a graph.

Theorem 1.4. [4] Let (X, d) be a complete metric space endowed with a graph $\mathrm{G}$ and $T: X \rightarrow X$ be a G-Kannan mapping. We suppose that:

(i) $\mathrm{G}$ is weakly $\mathrm{T}$-connected;

(ii) for any $\left(x_{n}\right)_{n \in N}$ in $\mathrm{X}$, if $x_{n} \rightarrow x$ and $\left(x_{n}, x_{n+1}\right) \in E(G)$ for $n \in N$ then

there is a subsequence $\left(x_{k_{n}}\right)_{n \in N}$ with $\left(x_{k_{n}}, x\right) \in E(G)$ for $n \in N$.

Then $T$ is a PO.

Samreen and Kamran [16] proved fixed point theorem for weakly contractive mappings on a metric space endowed with a graph.

Theorem 1.5. [16] Let $(X, d)$ be a completed metric space endowed with a graph $G$ and $f$ be a weakly G- contractive mapping from X into X. Suppose that the following condition holds.

(i) G satisfies property $\left(p^{\prime}\right)$,

(ii) there exist some $x_{0} \in X_{f}:=\{x \in X:(x, f x) \in E(G)\}$.

Then $\left.f\right|_{\left[x_{0}\right]_{\tilde{G}}}$ has a unique fixed point $\xi \epsilon\left[x_{0}\right]_{\tilde{G}}$ and $f^{n} y \rightarrow \xi$ for any $y \epsilon\left[x_{0}\right]_{\tilde{G}}$.

Aghaninas and Nourouzi [2] proved Banach and Kannan contraction in modular spaces with a graph.

Theorem 1.6. [2] Let $X$ be a $\rho$-complete modular space endowed with a graph $\mathrm{G}$ and the triple $(X, \rho, G)$. Moreover, this fixed point is unique if $k<\frac{1}{2}$ and $X$ satisfies the following condition For all $x, y \in X$, there exists a $z \in X$ such that $(x, z),(y, z) \in E(\tilde{G})$. Then a Kannan $\tilde{G}-\rho$ contraction $f: X \rightarrow X$ has a fixed point ifand only if $C_{f} \neq \varnothing$.

Alfuraidan [3] proved the contraction principle for mappings on a modular metric space with a graph.

Theorem 1.7. [3] Let $(X, \omega)$ be a modular metric space with a graph $G_{\omega}$. Suppose that $\omega$ is a convex regular modular metric which satisfies the $\Delta_{2}-$ type condition. Assume that $M=V\left(G_{\omega}\right)$ is a nonempty $\omega$ - bounded, $\omega$ - complete subset of $X_{\omega}$ and the triple $\left(M, d_{\omega}^{*}, G_{\omega}\right)$ has property (P) Let $T: M \rightarrow M$ be $G_{\omega}$-contraction map and $M_{T}:=$ $\left\{x \in M ;(x, T x) \in E\left(G_{\omega}\right)\right\}$.

If $\left(x_{0}, T\left(x_{0}\right)\right) \in E\left(G_{\omega}\right)$, then the following statement holds:

(i) For any $x \in M_{T},\left.T\right|_{[x]_{\widetilde{G_{\omega}}}}$ has a fixed point. 
Prerna Pathak, Aklesh Pariya, V. H. Badshah and Nirmala Gupta

(ii) If $G_{\omega}$ is weakly connected, then $\mathrm{T}$ has a fixed point in $\mathrm{M}$.

(iii) If $M^{\prime}:=\bigcup\left\{[x]_{\widetilde{G_{\omega}}}: x \in M_{T}\right\}$, then $\left.T\right|_{M^{\prime}}$ has a fixed point in $\mathrm{M}$.

\section{Basic definition and preliminaries}

Let $\mathrm{X}$ be a nonempty set. Throughout this paper for a function $\omega:(0, \infty) \times X \times X \rightarrow$ $(0, \infty)$ will be written as $\omega_{\lambda}(x, y)=\omega(\lambda, x, y)$ for all $\lambda>0$ and $x, y \in X$.

Definition 2.1. [5,6] Let $X$ be a non-empty set. A function $\omega:(0, \infty) \times X \times X \rightarrow[0, \infty]$ is said to be a metric modular on $X$ if it satisfies the following three axioms:

(i) given $x, y \in X, \omega_{\lambda}(x, y)=0$ for all $\lambda>0$ if and only if $x=y$;

(ii) $\quad \omega_{\lambda}(x, y)=\omega_{\lambda}(y, x)$ for all $\lambda>0$ and $x, y \in X$;

(iii) $\omega_{\lambda+\mu}(x, y) \leq \omega_{\lambda}(x, z)+\omega_{\mu}(z, y)$ for all $\lambda, \mu>0$ and $x, y, z \in X$.

If instead of (i), we have only the condition

$$
\omega_{\lambda}(x, x)=0 \text { for all } \lambda>0 \text { and } x \in X .
$$

Then $\omega$ is said to be a (metric) pseudo modular on X. A modular $\omega$ on $X$ is said to be regular if the following weaker version of (i) is satisfied:

$x=y$ if and only if $\omega_{\lambda}(x, y)=0$ for some $\lambda>0$.

Finally $\omega$ is said to be convex if for $\lambda, \mu>0$ and $\mathrm{x}, \mathrm{y}, \mathrm{z} \in \mathrm{X}$, it satisfies the inequality

$$
\omega_{\lambda+\mu}(x, y)=\frac{\lambda}{\lambda+\mu} \omega_{\lambda}(x, z)+\frac{\mu}{\lambda+\mu} \omega_{\mu}(z, y) .
$$

Note that for a pseudo modular $\omega$ on a set $\mathrm{X}$ and any $x, y \in X$, the function $\lambda \rightarrow \omega_{\lambda}(x, y)$ is non increasing on $(0, \infty)$. Indeed, if $0<\mu<\lambda$, then

$$
\omega_{\lambda}(x, y) \leq \omega_{\lambda-\mu}(x, x)+\omega_{\mu}(x, y)=\omega_{\mu}(x, y)
$$

Definition 2.2. Let $X_{\omega}$ be a modular metric space.

(1) The sequence $\left(x_{n}\right)_{n \in N}$ in $X_{\omega}$ is said to be convergent to $x \in X_{\omega}$ if

$$
\omega_{\lambda}\left(x_{n}, x\right) \rightarrow 0 \text { as } n \rightarrow \infty \text { for all } \lambda>0 \text {. }
$$

(2) The sequence $\left(x_{n}\right)_{n \in N}$ in $X_{\omega}$ is said to be Cauchy if

$$
\omega_{\lambda}\left(x_{m}, x_{n}\right) \rightarrow 0 \text { asm } n \rightarrow \infty \text { for all } \lambda>0 \text {. }
$$

(3) A subset $\mathrm{C}$ of $X_{\omega}$ is said to be closed if the limit of the convergent sequence of $\mathrm{C}$ always belong to $\mathrm{C}$.

(4) A subset $\mathrm{C}$ of $X_{\omega}$ is said to be complete if any Cauchy sequence in $\mathrm{C}$ is a convergent sequence and its limit in $\mathrm{C}$.

(5) A subset $\mathrm{C}$ of $X_{\omega}$ is said to be bounded if for all $\lambda>o$

$$
\delta_{\omega}(C)=\sup \left\{\omega_{\lambda}(x, y) ; x, y \in C\right\}<\infty .
$$

We will use following notations and terminology of graph theory (see [3]) related to the rest of our result.

Let $(X, \omega)$ be a modular metric space and $M$ be a non empty subset of $X_{\omega}$. Let $\Delta$ denote the diagonal of the Cartesian product $M \times M$. Consider a directed graph $G_{\omega}$ such that the set $V\left(G_{\omega}\right)$ of its vertices coincide with $\mathrm{M}$, and the set $E\left(G_{\omega}\right)$ of its edges contain all loops, i.e. $E\left(G_{\omega}\right) \supseteq \Delta$. We assume $G_{\omega}$ has no parallel edges (arcs), so we can identify $G_{\omega}$ with the pair $\left(V\left(G_{\omega}\right), E\left(G_{\omega}\right)\right)$. Our graph theory notation and terminology are standard and can be found in all graph theory books, like [14]. Moreover, we may treat $G_{\omega}$ as a weighted graph (see [10]) by assigning to each edge the distance between its vertices. 
Fixed Point Theorems for Kannan Contractions and Weakly Contractive Mappings on a Modular Metric Space Endowed with a Graph

By $\mathrm{G}^{-1}$ we denote the conversion of a graph $G$, i.e., the graph obtained from $G$ by reversing the direction of edges. Thus we have

$$
E\left(\mathrm{G}^{-1}\right)=\{(y, x) \mid(x, y) \in E(G)\} .
$$

A diagraph $\mathrm{G}$ is called an oriented graph if whenever $(u, v) \in E(G)$, then $(v, u) \notin$ $E(G)$. The letter $\tilde{G}$ denotes the undirected graph obtain from $\mathrm{G}$ by ignoring the direction of edges.

Actually, it will be more convenient for us to treat $\tilde{G}$ as a directed graph for which the set of its edges is symmetric. Under this convention, $E(\tilde{G})=E(G) \cup E\left(\mathrm{G}^{-1}\right)$.

We call $\left(V^{\prime}, E^{\prime}\right)$ a sub graph of $V^{\prime} \subseteq V(G), E^{\prime} \subseteq E(G)$, and for any edge $(x, y) \in E^{\prime}, x, y \in V^{\prime}$.

If $x$ and $y$ are vertices in a graph $\mathrm{G}$, then a (directed) path in $\mathrm{G}$ from $x$ to $y$ of length $\mathrm{N}$ is a sequence $\left(x_{i}\right)_{i=1}^{N}$ of $N+1$ vertices such that $x_{0}=x, x_{N}=y$ and $\left(x_{n-1}, x_{n}\right) \in E(G)$ for $i=1, \ldots, N$. A graph $\mathrm{G}$ is connected if there is a directed path between any two vertices. $\mathrm{G}$ is a weakly connected if $\tilde{G}$ is connected. If $\mathrm{G}$ is such that $E(G)$ is symmetric and $x$ is a vertex in $\mathrm{G}$, then the sub graph $G_{x}$ consisting of all edges and vertices which are contained in some path beginning at $x$ is called the component of G containing $x$. In this case $V\left(G_{x}\right)=[x]_{G}$, where $[x]_{G}$ is the equivalence class of the following relation $\mathcal{R}$ defined on $V(G)$ by the rule:

$y \mathcal{R} z$ if there is a (directed) path in $\mathrm{G}$ from $y$ to $z$.

Clearly $G_{x}$ is connected.

Definition 2.3. [3] Let $(X, \omega)$ be a modular metric space and $M$ be a non empty subset of $X_{\omega}$. A mapping $T: M \rightarrow M$ is called

(i) $G_{\omega}$ - contraction if $\mathrm{T}$ preserve edges of $G_{\omega}$, i.e.,

$$
\forall x, y \in M\left((x, y) \in E\left(G_{\omega}\right) \Rightarrow(T(x), T(y)) \in E\left(G_{\omega}\right)\right),
$$

and if there exists a constant $\alpha \in[0,1)$ such that

$$
\omega_{1}(T(x), T(y)) \leq \alpha \omega_{1}(x, y) \text { for any }(x, y) \in E\left(G_{\omega}\right) .
$$

(ii) $(\varepsilon, \alpha)-G_{\omega}$-uniformly locally contraction if T preserve edges of $G_{\omega}$ and there exists a Constant $\alpha \in[0,1)$ such that for any $(x, y) \in E\left(G_{\omega}\right)$

$\omega_{1}(T(x), T(y)) \leq \alpha \omega_{1}(x, y)$ whenever $\omega_{1}(x, y)<\varepsilon$.

Definition 2.4. [3] A point $x \in M$ is called a fixed point of $\mathrm{T}$ whenever $x=T(x)$. The set of fixed points of $\mathrm{T}$ will be denoted by Fix(T).

Now we introduce the $G_{\omega}$ Kannan contraction and weakly $G_{\omega}$ contractive mappings in a modular metric space endowed with a graph as follows.

Definition 2.5. Let $(X, \omega)$ be a modular metric space with a graph $G_{\omega}$. Amapping $T: M \rightarrow M$ is called

(1) $G_{\omega}$ - Kannan contraction if $\mathrm{T}$ preserve the edges of $G_{\omega}$, i.e., for all $x, y \in M\left((x, y) \in E\left(G_{\omega}\right) \Rightarrow(T x, T y) \in E\left(G_{\omega}\right)\right)$

and if there exists positive number $k \in\left(0, \frac{1}{2}\right)$ such that 
Prerna Pathak, Aklesh Pariya, V. H. Badshah and Nirmala Gupta

$\omega_{\lambda}(T x, T y) \leq k\left(\omega_{\lambda}(T x, x)+\omega_{\lambda}(T y, y)\right)$ for any $x, y \in M$ with $(x, y) \in E\left(G_{\omega}\right)$.

weakly $G_{\omega}$ contractive if T preserve the edges of $G_{\omega}$,

i.e., for all $x, y \in M\left((x, y) \in E\left(G_{\omega}\right) \Rightarrow(T x, T y) \in E\left(G_{\omega}\right)\right)$

and $\omega_{\lambda}(T x, T y) \leq \omega_{\lambda}(x, y)-\psi\left(\omega_{\lambda}(x, y)\right)$

whenever $\psi:[0, \infty) \rightarrow[0, \infty)$ is continuous non decreasing such that $\psi$ is positive on $(0, \infty)$ and $\psi(0)=0$.

Our first result can be seen as an extension of Jachymski [9] fixed point theorems to modular metric spaces. As Jachymski [8] did, we introduce the following property.

We say that the triple $\left(M, d_{\omega}^{*}, G_{\omega}\right)$ has property $(\mathrm{P})$ if

(P) For any sequence $\left\{x_{n}\right\}_{n \in N}$ in M, if $x_{n} \rightarrow x$ as $n \rightarrow \infty$ and $\left(x_{n}, x_{n+1}\right) \in E\left(G_{\omega}\right)$, then $\left(x_{n}, x\right) \in E\left(G_{\omega}\right)$, for all $\mathrm{n}$.

Note that property $(\mathrm{P})$ is precisely the Nieto et al. [14] hypothesis relaxing continuity assumption as in Theorem $1.2((2)$ and (3)) rephrased in terms of edges.

Lemma 2.1. [16] Let $(X, d)$ be a metric space and $T: X \rightarrow X$ be a weakly $G$-contractive map. Then for any $x \in X$ and $y \in[x]_{\tilde{G}}$ we have

$$
\lim _{n \rightarrow \infty} d\left(T^{n} x, T^{n} y\right)=\lim _{n \rightarrow \infty} r\left(T^{n} x, T^{n} y\right)=0 .
$$

Proposition 2.2. [16] Let $(X, d)$ be a metric space and $T$ be a weakly $G$-contractive mapping from $\mathrm{X}$ into $\mathrm{X}$. Let there exist $x_{0} \in X$ such that $T x_{0} \in\left[x_{0}\right]_{\tilde{G}}$ then the sequence $\left\{T^{n} x_{0}\right\}$ is Cauchy.

\section{Main results}

Theorem 3.1. Let $(X, \omega)$ be a modular metric space with a graph $G_{\omega}$. Suppose that $\omega$ is a convex regular modular metric which satisfies the $\Delta_{2}$ - type condition. Assume that $M=V\left(G_{\omega}\right)$ is a nonempty $\omega$ - bounded, $\omega$-complete subset of $X_{\omega}$ and the triple $\left(M, d_{\omega}^{*}, G_{\omega}\right)$ has property $(\mathrm{P})$. Let $T: M \rightarrow M$ be Kannan contraction map and $M_{T}:=$ $\left\{x \in M ;(x, T x) \in E\left(G_{\omega}\right)\right\}$.

If $\left(x_{0}, T\left(x_{0}\right)\right) \in E\left(G_{\omega}\right)$, then the following statements hold:

(i) For any $x \in M_{T},\left.T\right|_{[x]_{\widetilde{G_{\omega}}}}$, has a fixed point.

(ii) If $G_{\omega}$ is weakly connected, then $\mathrm{T}$ has a fixed point in $\mathrm{M}$.

(iii) If $M^{\prime}=\bigcup\left\{[x]_{\widetilde{G_{\omega}}}: x \in M_{T}\right\}$, then $\left.T\right|_{M^{\prime}}$ has a fixed point in $\mathrm{M}$.

Proof (i): As $\left(x_{0}, T\left(x_{0}\right)\right) \in E\left(G_{\omega}\right)$ and $\left(y_{0}, T\left(y_{0}\right)\right) \in E\left(G_{\omega}\right)$ then $x_{0}, y_{0} \in M_{T}$. Since $\mathrm{T}$ is a Kannan-contraction, there exists a constant $k \in\left(0, \frac{1}{2}\right)$ such that $\left(T\left(x_{0}\right), T\left(y_{0}\right)\right) \in E\left(G_{\omega}\right)$ and $\omega_{1}\left(T x_{0}, T y_{0}\right) \leq k\left[\omega_{1}\left(x_{0}, T x_{0}\right)+\omega_{1}\left(y_{0}, T y_{0}\right)\right]$

By induction we can construct a sequence $\left\{x_{n}\right\}$ such that $x_{n+1}=T x_{n}$ and $\left(x_{n}, x_{n+1}\right) \in E\left(G_{\omega}\right)$

$$
\begin{gathered}
\omega_{1}\left(x_{n+1}, x_{n}\right)=\omega_{1}\left(T x_{n}, T x_{n-1}\right) \\
\omega_{1}\left(x_{n+1}, x_{n}\right) \leq k\left[\omega_{1}\left(T x_{n}, x_{n}\right)+\omega_{1}\left(T x_{n-1}, x_{n-1}\right)\right] \\
\leq k\left[\omega_{1}\left(x_{n+1}, x_{n}\right)+\omega_{1}\left(x_{n}, x_{n-1}\right)\right] \\
(1-k) \omega_{1}\left(x_{n+1}, x_{n}\right) \leq k \omega_{1}\left(x_{n}, x_{n-1}\right) \\
\omega_{1}\left(x_{n+1}, x_{n}\right) \leq \frac{k}{(1-k)} \omega_{1}\left(x_{n}, x_{n-1}\right) \text { where } \alpha=\frac{k}{(1-k)}<1 \\
\omega_{1}\left(x_{n+1}, x_{n}\right) \leq \alpha \omega_{1}\left(x_{n}, x_{n-1}\right)
\end{gathered}
$$


Fixed Point Theorems for Kannan Contractions and Weakly Contractive Mappings on a Modular Metric Space Endowed with a Graph

So by induction, we construct a sequence $\left\{x_{n}\right\}$ such that $\left(x_{n+1}, x_{n}\right) \in E\left(G_{\omega}\right)$ and $\omega_{1}\left(x_{n+1}, x_{n}\right) \leq \alpha^{n} \omega_{1}\left(x_{0}, x_{1}\right)$ for any $n \geq 1$. Since $M$ is $\omega$-bounded, we have

for any $n \geq 1$. Then by lemma 2.2 .

$$
\omega_{1}\left(x_{n+1}, x_{n}\right) \leq \delta_{\omega}(M) k^{n}
$$

$\Longrightarrow\left\{x_{n}\right\}$ is $\omega$-Cauchy. Since $M$ is $\omega$-Complete, therefore $\left\{x_{n}\right\}$ is $\omega$ - convergence to some point $\epsilon M$.

By property $(\mathrm{P}),\left(x_{n}, x\right) \in E\left(G_{\omega}\right)$ for all $n$ and hence

$$
\begin{aligned}
& \omega_{1}\left(x_{n+1}, T(x)\right)=\omega_{1}\left(T x_{n}, T x\right) \\
& \leq k\left(\omega_{1}\left(T x_{n}, x_{n}\right)+\omega_{1}(T x, x)\right)
\end{aligned}
$$

Taking limit $n \rightarrow \infty$ both sides we get

$$
\omega_{1}(x, T x) \leq k\left(\omega_{1}(x, x)+\omega_{1}(T x, x)\right)
$$

i.e. $\left.\omega_{1}(x, T x) \leq k \omega_{1}(T x, x)\right)$ which is a contradiction.

Hence $\omega_{1}(x, T x)=0$.

Therefore $x=T x$.

i.e. $x$ is a fixed point of $\mathrm{T}$.

As $\left(x_{0}, x\right) \in E\left(G_{\omega}\right)$, we have $x \in\left[x_{0}\right]_{\widetilde{G_{\omega}}}$.

Uniqueness. Let $x$ and $\mathrm{y}$ be two fixed point of $\mathrm{T}$.

Consider $\omega_{1}(x, y)=\omega_{1}(T x, T y) \leq k\left[\omega_{1}(x, T x)+\omega_{1}(y, T y)\right]$

This gives

$$
\leq k\left[\omega_{1}(x, x)+\omega_{1}(y, y)\right]
$$

Hence point is unique.

$$
\omega_{1}(x, y)=0 \Rightarrow x=y \text {. }
$$

(ii) Since $M_{T} \neq \varnothing$, there exists an $x_{0} \in M_{T}$ and since $G_{\omega}$ is weakly connected, then $\left[x_{0}\right]_{\widetilde{G_{\omega}}}=M$ and by $\mathrm{M}$ and by (i), mapping $\mathrm{T}$ has a fixed point.

(iii) It follows easily from (i) and (ii).

Theorem 3.2. Let $(X, \omega)$ be a modular metric space with a graph $G_{\omega}$. Suppose that $\omega$ is a convex regular modular metric which satisfies the $\Delta_{2}$ - type condition. Assume that $M=V\left(G_{\omega}\right)$ is a nonempty $\omega$ - bounded, $\omega$ - complete subset of $X_{\omega}$ and the triple $\left(M, d_{\omega}^{*}, G_{\omega}\right)$ has property $(\mathrm{P})$. Let $T: M \rightarrow M$ be weak contraction mapping and $M_{T}:=$ $\left\{x \in M ;(x, T x) \in E\left(G_{\omega}\right)\right\}$.

If $\left(x_{0}, T\left(x_{0}\right)\right) \in E\left(G_{\omega}\right)$, then the following statements hold:

(iv) For any $x \in M_{T},\left.T\right|_{[x]_{\widetilde{G_{\omega}}}}$, has a fixed point.

(v) If $G_{\omega}$ is weakly connected, then $\mathrm{T}$ has a fixed point in $\mathrm{M}$.

(vi) If $M^{\prime}=\bigcup\left\{[x]_{\widetilde{G_{\omega}}}: x \in M_{T}\right\}$, then $\left.T\right|_{M^{\prime}}$ has a fixed point in M.

Proof: As $\left(x_{0}, T\left(x_{0}\right)\right) \epsilon E\left(G_{\omega}\right)$ and $\left(y_{0}, T\left(y_{0}\right)\right) \epsilon E\left(G_{\omega}\right)$ then $x_{0}, y_{0} \in M_{T}$. Since $\mathrm{T}$ is a weak contraction, there exists a constant $k \in\left(0, \frac{1}{2}\right)$ such that $\left(T\left(x_{0}\right), T\left(y_{0}\right)\right) \in E\left(G_{\omega}\right)$ and

$$
\omega_{1}\left(T x_{0}, T y_{0}\right) \leq \omega_{1}\left(x_{0}, y_{0}\right)-\Psi\left(\omega_{1}\left(x_{0}, y_{0}\right)\right)
$$

By induction we can construct a sequence $\left\{x_{n}\right\}$ such that $x_{n+1}=T x_{n}$ and

Similarly

$$
\begin{gathered}
\left(x_{n}, x_{n+1}\right) \in E\left(G_{\omega}\right) \omega_{1}\left(x_{n+1}, x_{n}\right)=\omega_{1}\left(T x_{n}, T x_{n-1}\right) \\
\omega_{1}\left(x_{n+1}, x_{n}\right) \leq \omega_{1}\left(x_{n}, x_{n-1}\right)-\psi\left(\omega_{1}\left(x_{n}, x_{n-1}\right)\right) \\
\omega_{1}\left(x_{n+1}, x_{n}\right) \leq \omega_{1}\left(x_{n}, x_{n-1}\right)
\end{gathered}
$$


Prerna Pathak, Aklesh Pariya, V. H. Badshah and Nirmala Gupta

Hence in general

$$
\begin{gathered}
\omega_{1}\left(x_{n+2}, x_{n+1}\right)=\omega_{1}\left(T x_{n+1}, T x_{n}\right) \\
\omega_{1}\left(x_{n+2}, x_{n+1}\right) \leq \omega_{1}\left(x_{n+1}, x_{n}\right)-\Psi\left(\omega_{1}\left(x_{n+1}, x_{n}\right)\right) \\
\omega_{1}\left(x_{n+2}, x_{n+1}\right) \leq \omega_{1}\left(x_{n+1}, x_{n}\right) \\
\omega_{1}\left(x_{n+3}, x_{n+2}\right)=\omega_{1}\left(T x_{n+2}, T x_{n+1}\right) \\
\omega_{1}\left(x_{n+3}, x_{n+2}\right) \leq \omega_{1}\left(x_{n+2}, x_{n+1}\right)-\Psi\left(\omega_{1}\left(x_{n+2}, x_{n+1}\right)\right) \\
\omega_{1}\left(x_{n+3}, x_{n+2}\right) \leq \omega_{1}\left(x_{n+2}, x_{n+1}\right)
\end{gathered}
$$

$$
\omega_{1}\left(x_{i+1}, x_{n}\right) \leq \omega_{1}\left(x_{i}, x_{i-1}\right)-\Psi\left(\omega_{1}\left(x_{i}, x_{i-1}\right) ; \quad \forall i=1,2,3 \ldots n\right.
$$

Since $\Psi$ is non decreasing and this shows that $\left\{x_{i}\right\}_{i=1}^{n}$ is a $\omega$-cauchy sequence

$$
\omega_{1}\left(x_{n+1}, x_{n}\right) \leq \omega_{1}\left(x_{n}, x_{n-1}\right) \leq \cdots \leq \omega_{1}\left(x_{1}, x_{0}\right)
$$

Since $\omega_{1}\left(x_{n+1}, x_{n}\right)$ is non increasing sequence of non-negative real number bounded below by 0 , thus convergent.

Taking limit as $\mathrm{n} \rightarrow \infty$,

$\lim _{n \rightarrow \infty} \omega_{1}\left(x_{n+1}, x_{n}\right)=0$;

$\forall i=1,2,3 \ldots n$.

Uniqueness: Let $x$ and $\mathrm{y}$ be two fixed point of T.

Consider $\left.\omega_{1}(x, y)=\omega_{1}(T x, T y) \leq \omega_{1}(x, y)-\psi \omega_{1}(x, y)\right]$

This gives

Hence point is unique.

$$
\omega_{1}(x, y)=0 \Rightarrow x=y .
$$

(ii) Since $M_{T} \neq \varnothing$, there exists an $x_{0} \in M_{T}$ and since $G_{\omega}$ is weakly connected, then $\left[x_{0}\right]_{\widetilde{G_{\omega}}}=M$ and by $\mathrm{M}$ and by (i), mapping $\mathrm{T}$ has a fixed point.

(iii) It follows easily from (i) and (ii).

\section{REFERENCES}

1. A.N.Abdou and M.A.Khamsi, Fixed points of multi valued contraction mappings in modular metric spaces, Fixed Point Theory Appl., 249 (2014). doi:10.1186/16871812-2014-249.

2. A.Aghanians and K.Nourouzi, Fixed point for Banach and Kannan contractions in modular spaces with a graph, Int. J. Nonlinear Anal. Appl., 5(2) (2014) 50-59.

3. M.R.Alfuraidan, The contraction principle for mappings on a modular metric space with a graph, Fixed Point Theory and Applications (2015) 2015:46 DOI 10.1186/s13663-015-0296-3

4. F.Bojor, Fixed points of Kannan mappings in metric spaces endowed with a graph, An. St. Univ. Ovidius Constanta, 20(1) (2012) 31-40.

5. V.V.Chistyakov, Modular metric spaces, I, basic concepts, Nonlinear Anal., 72 (1) (2010) 1-14.

6. V.V.Chistyakov, Modular metric spaces, II, Application to superposition operators, Nonlinear Anal., 72(1) (2010) 15-30.

7. A.Granas and J.Dugundji, Fixed Point Theory, Springer, New York (2003).

8. J.Jachymski, Order-theoretic aspects of metric fixed point theory, Handbook of Metric Fixed Point Theory (eds., W. A. Kirk and B. Sims), 613-641, Kluwer Acad. Publ.,Dordrecht, 2001. MR1904289 (2003f:54094).

9. J.Jachymski, The contraction principle for mappings on a metric space with graph, Proc. Am. Math. Soc., (2008) 1359-1373.

10. R.Johnsonbaugh, Discrete Mathematics, Prentice Hall, New York (1997). 
Fixed Point Theorems for Kannan Contractions and Weakly Contractive Mappings on a Modular Metric Space Endowed with a Graph

11. S.Koshi and T.Shimogaki, On F-norms of quasi-modular spaces, J Fac Sci Hokkaido Univ Ser I, 15(3-4) (1961) 202-218.

12. J.Musielak and W.Orlicz, Some remarks on modular spaces, Bull Acad Polon Sci Sr Sci Math Astron Phys., 7 (1959) 661-668.

13. H.Nakano, Modulared Semi-Ordered Linear Spaces, i+288 PP. Maruzen, Tokyo (1950).

14. J.J.Nieto, R.L.Pouso and R.Rodriguez-Lopez, Fixed point theorems in ordered abstract spaces, Proc. Am. Math. Soc., 135 (2007) 2505-2517.

15. A.C.M.Ran, M.C.B.Reurings, A fixed point theorem in partially ordered sets and some applications to matrix equations, Proc. Am. Math. Soc., 132 (2004) 1435-1443.

16. M.Samreen and T.Kamran, Fixed point theorems for weakly contractive mappings on a metric space endowed with a graph, Filomat, 28(3) (2014) 441-450.

17. S.Yamamuro, On conjugate spaces of Nakano spaces, Trans Amer Math Soc., 90 (1959) 291-311. Doi: 10.1090/S0002-9947-1959-0132378-1. 